\title{
Why choose 3D bioprinting? Part III: printing in vitro 3D models for drug screening
}

\author{
Yong $\mathrm{He}^{1}$. Jing $\mathrm{Nie}^{1} \cdot$ Mingjun $\mathrm{Xie}^{1} \cdot \mathrm{Qing} \mathrm{Gao}^{1}$
}

Published online: 4 April 2020

(c) Zhejiang University Press 2020

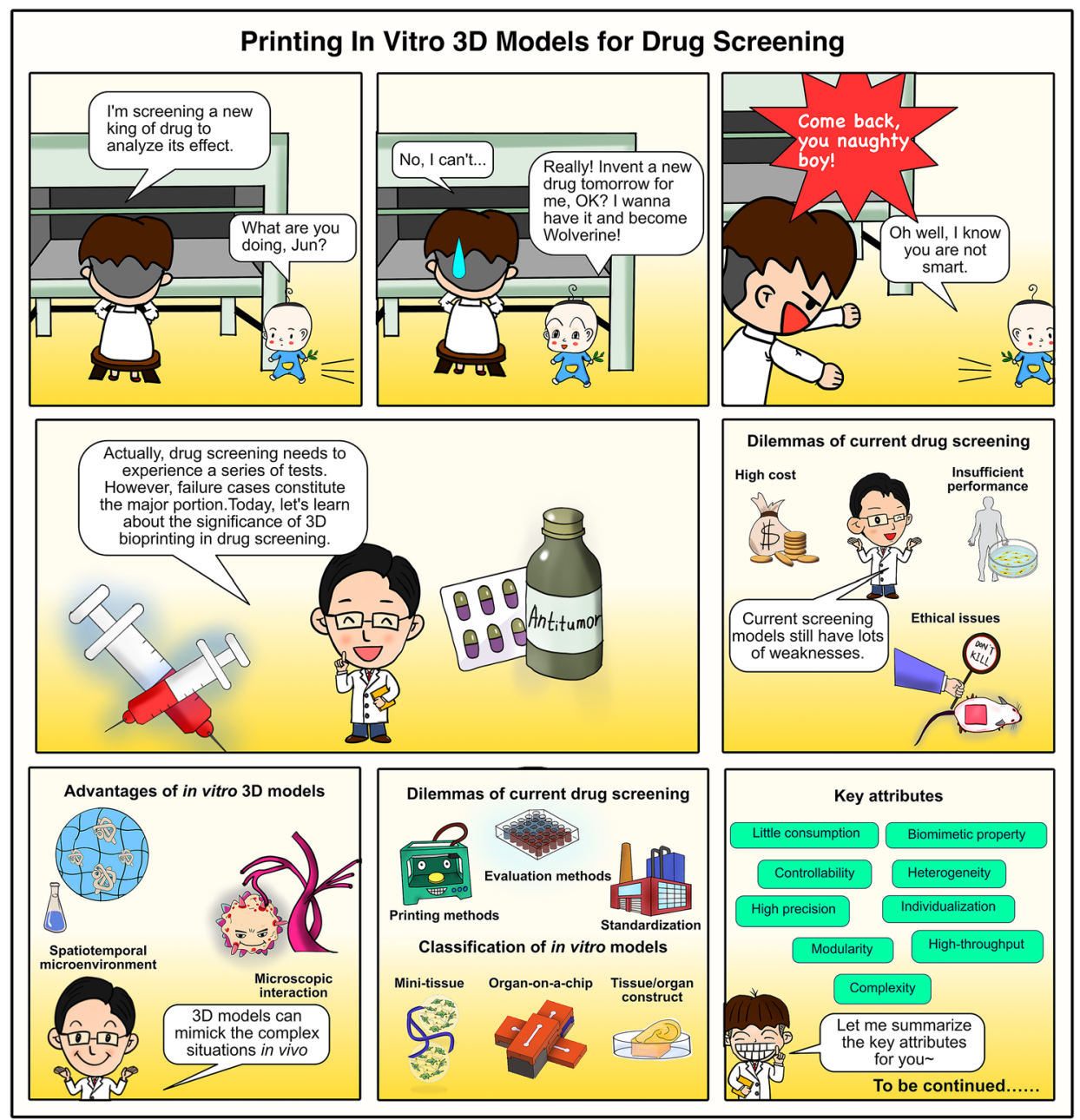

$凶$ Yong He

yongqin@zju.edu.cn

1 State Key Laboratory of Fluid Power and Mechatronic Systems, School of Mechanical Engineering, Zhejiang University, Hangzhou 310027, China 


\section{Dilemmas of current drug screening}

Recently, multiple technological advancements have been involved in the process of drug research and discovery. In addition, the spending on the relevant projects has doubled in the last 20 years, cumulatively increasing 28\% from 2014 to 2016 . However, these improvements have not led directly to an obvious increase in feasible drug candidates that successfully passed the clinical trials. According to the increased spending and stagnant clinical success rate of $10-20 \%$, the efficiency of this project drastically decreased from around 40 drugs per billion in 1950 to 0.6 drugs in 2012. The estimated costs for different drugs vary from 160 Mio. USD to over 2.5 billion USD, among which the failure cases constitute the major portion. Furthermore, 15-30\% failures are attributed to safety and efficacy reasons, while $>60 \%$ of failures are caused by toxicity issues in early phase [1]. These factors collectively led to the stuck of drug discovery process.

It is necessary to conduct a series of screening procedures in aspects of activity, toxicity, metabolism, efficacy, side effect, dose response before clinical trials. The main bottleneck is lacking an accurate platform for the individualized assessment of the drug candidates. What's more, the actual action of certain drug involves several interconnected organs. In order to realize more precision drug screening, it is crucial to construct corresponding models as accurate as possible.

For a long time, pre-clinical validations are mainly based on conventional $2 \mathrm{D}$ cell-monolayer culture and animal experiments [2-4]. Conventional cell-monolayer planar culture model largely varies from the complicated in vivo microenvironment, lacking necessary interaction and physiological activity. Also, animal experiments are time-consuming and ethically restricted, making them a high-cost proposition. What's more, animal models are invalid to reproduce human physiological situation or accurately predict clinical reaction.

The insufficient performance, limited flexibility, ethical issues, as well as high cost of existing drug screening strategies have led to a growing demand on better in vitro models [5] that can represent the complication of human tissues/organs and in vivo physiological/pathological conditions to complement the existing methods for drug evaluation. Researchers are seeking to build more precise organ models and co-culture models for better evaluation of drug candidates. The possibility of precise detection and prediction on both efficacy and toxicity depends on the availability of robust assay models that could reproduce in vivo condition targeting at individual patient.

\section{Advantages of in vitro 3D models}

An ideal in vitro model displays better capability of reproducing in vivo physiological/pathological condition and recreating the morphology and function of human tissue/organ, contributing to the improvement of drug screening efficiency.

The in vitro 3D models can act as an alternative to the traditional drug-screening models to a certain extent and replace a portion of high-cost in vivo experiments, helping to decrease the time and cost for new drug development. In addition, it can mimic the in vivo environment better, with more flexibility and diversity, which can be applied in wide biomedical fields.

\section{Printing of in vitro 3D models}

Defined as the deposition of pre-prepared bioink (biomaterials or cells) based on the principle of additive manufacturing, $3 \mathrm{D}$ bioprinting technology has advanced to enable the precise distribution of a specific type of cell or biomaterial in a target area. It can simultaneously construct the reaction container, entire 3D structure, endogenous cell-cell/cell-matrix interaction, and in vivo microenvironment with inherent spatial and chemical complexity.

Recently, 3D bioprinting has advanced to enable the construction of cell-laden structures through the on-demand distribution of biomaterials and cells, as well as the precise manipulation of chemical environment through the design of specific architecture. It has enabled the simultaneous construction of the macroscopic entity, microscopic interaction, and spatiotemporal microenvironment. Along with its inherent superiorities of flexibility and versatility, 3D bioprinting is becoming a promising scheme for the construction of personalized in vitro models with medical significance, offering exciting prospects for precision medicine [6].

In order to obtain robust models for reliable results, cell types and sources, printing bioinks, as well as printing strategies have to be seriously selected and modified. It is promising for overcoming the current challenges of tissue engineering and drug development with printed personalized complex tissue/organ models. Different types of organ vary in composition, structure, and function, calling demands on corresponding cell sources, matrix materials, processing approaches, and culture conditions. There exists some common printing strategies and construct forms with correspondence to different organ types. 


\section{Classification of in vitro models}

The in vitro models have experienced a meaningful revolution in forms and functions: mini-tissue, organ-on-a-chip, and tissue/organ construct.

Mini-tissue 3D mini-constructs in forms of sphere, fiber, or other geometries can be printed based on common printing techniques, including inkjet-based, extrusion-based, and light-assisted bioprinting based on various cell sources and biomaterials. These models are suitable for the simulation of functional units with relative simple composition and independent operation, which can facilitate high-throughput testing with a low dose. As a representative model, hydrogel microspheres have been widely applied in cell therapy, local tissue filling, as well as high-throughput drug screening [7]. In addition, cell-laden hydrogel microfibers with specific morphology and function have been applied as biomimicking models of a set of strip structures, such as muscle, nerve, and vessel [8].

Organ-on-a-chip Through the introduction of the microfluidic technology, a set of organ-on-a chip models have been proposed to mimic the in vivo microenvironment and the main function of specific tissue/organ [9]. External stimuli can be applied through the microfluidic means, serving as a platform for in situ detection. 3D bioprinting technology has been gradually involved in the building of organ-on-a-chip devices with its excellent customization and cell compatibility [6]. A set of customized microfluidic systems have been constructed with biomaterials through 3D bioprinting, upon which some meaningful cells are loaded and conventional reactions are conducted. Inspired by the concept of modularity, traditional organ-on-a chip has been divided into several functional units with simple structures. The whole process is largely simplified in terms of design, manufacturing, and modification. Furthermore, the interaction and crosstalk between multiple organs can be properly modeled through the connection of different modules with microfluidic means.

Tissuelorgan construct With the ability of constructing cell-laden structures, 3D bioprinting has been further applied in the generation of tissue/organ constructs with inner channel network. On the basis of the materials' good biological performance and ECM-mimicking property, cells can be located both in the material bulk as 3D tissue and on the channel surface to mimic vascular structure [10]. Variety of 3D bioprinting strategies have been employed in engineering 3D tissue/organ constructs with inner vascular network, including coaxial printing, projection-based printing, as well as the combination of $3 \mathrm{D}$ bioprinting and sacrificing of templates. The generation of large-scale vascularized tissue/organ models advances the development of precision medicine in terms of tissue regen- eration, organ transplantation, as well as drug screening [11].

\section{Current challenge}

Although 3D bioprinting has been broadly applied to constructing in vitro models, non-negligible challenges still exist.

- Printing methods The experimental protocols for drug evaluation have to meet the demands of high-throughput and standardization. In aspects of the printing process, researchers are faced with challenges in the printing resolution, printing efficiency, as well as repeatability. On the other hand, reliable cell sources, biomaterials, and induction condition are indispensable for the achievement of effective models with corresponding morphologies and functions for drug screening.

- Evaluation methods The transition from 2D models towards 3D models is bringing about a set of incompatibility of the systematic cell evaluation and testing. The chemical detection represented by the kit and visual inspection represented by the confocal is initially designed for the purpose of $2 \mathrm{D}$ assay. In order to be adapted to $3 \mathrm{D}$ models, technological advances in imaging techniques and analytical tools may also be in pressing demand for subsequent analysis and evaluation. In addition, a series of promising protocols should be modified or newly developed in order to realize the direct detection process on 3D models, such as visual inspection, microscopy observation, pathogenic detection, immunological detection, biochemical testing, and molecular biology detection.

- Standardization In the process of industrialization, sample consistency and process stability are of extreme importance for reliable results and standard products. However, relevant research is still in its infancy, and it is nearly an empty field about the normalized printing structures, standardized process, and protocol for the evaluation of the in vitro $3 \mathrm{D}$ models.

In additional to challenges in the technical level, further applications bring about a set of issues to be addressed. In aspects of the specific application scenario, the interindividual difference brings high complexity and variability, calling for individualized models containing unique cell sources and matrix properties [12]. In order to better mimic the further process of transplantation and clinical trial [13], during which the in vivo immunity and metabolic system is bringing challenges and causing effects, an interconnected dynamic culture system could be applied to the in vitro models. 


\section{Key attributes}

The distinct advantages of the 3D bioprinting technology facilitate the realization of the modular and standard construction of in vitro models via a high-throughput method for personalized drug screening. Corresponding merits of 3D bioprinted in vitro models are listed as follows:

- Biomimetic property

- Modularity

- Little consumption

- Individualization

- Controllability

- High-throughput

- Heterogeneity

- Complexity

- High precision

\section{Conclusion}

In order to relieve the grim situation of drug discovery, in vitro model is utilized as a promising alternative for conventional 2D cell culture and experimental animal due to its excellent in vivo mimicking characteristic. As an advanced technology for the manipulation of biomaterials and cells, 3D bioprinting has been extensively applied in the construction of multiple in vitro models for biomedical applications. The bioprinted in vitro models experienced a gradual improvement in the developing process, during which a set of in vitro models are derived with unique transcendence. The introduction of 3D bioprinting technology to the construction of in vitro models for drug screening has exhibited a series of unique key attributes as well as inevitable relevant challenges.

\section{Compliance with ethical standards}

Conflict of interest The authors declare that they have no conflict of interest.

Human and animal rights This article does not contain any studies with human or animal subjects performed by any of the authors.

\section{References}

1. Shen JX, Youhanna S, Zandi Shafagh R, Kele J, Lauschke VM (2002) Organotypic and microphysiological models of liver, gut, and kidney for studies of drug metabolism, pharmacokinetics, and toxicity. Chem Res Toxicol 33:38-60

2. Ashby M, Rine J (1996) Methods for drug screening. Google patents

3. Drews J (2000) Drug discovery: a historical perspective. Science 287:1960-1964

4. Greek R, Menache A (2013) Systematic reviews of animal models: methodology versus epistemology. Int J Med Sci 10:206

5. Dambach DM, Andrews BA, Moulin F (2005) New technologies and screening strategies for hepatotoxicity: use of in vitro models. Toxicol Pathol 33:17-26

6. Yi H-G, Lee H, Cho D-W (2017) 3D printing of organs-on-chips. Bioengineering 4:10

7. Xie M, Gao Q, Zhao H, Nie J, Fu Z, Wang H et al (2019) Electroassisted bioprinting of low-concentration GelMA microdroplets. Small 15:1804216

8. Shao L, Gao Q, Zhao H, Xie C, Fu J, Liu Z et al (2018) Fiber-based mini tissue with morphology-controllable GelMA microfibers. Small 14:1802187

9. Huh D, Torisawa Y-S, Hamilton GA, Kim HJ, Ingber DE (2012) Microengineered physiological biomimicry: organs-on-chips. Lab Chip 12:2156-2164

10. Richards D, Jia J, Yost M, Markwald R, Mei Y (2017) 3D bioprinting for vascularized tissue fabrication. Ann Biomed Eng 45:132-147

11. Nie J, Gao Q, Xie C, Lv S, Qiu J, Liu Y et al (2020) Construction of multi-scale vascular chips and modelling of the interaction between tumours and blood vessels. Mater Horiz 7:82-92

12. Rangarajan A, Hong SJ, Gifford A, Weinberg RA (2004) Speciesand cell type-specific requirements for cellular transformation. Cancer Cell 6:171-183

13. Force T, Kolaja KL (2011) Cardiotoxicity of kinase inhibitors: the prediction and translation of preclinical models to clinical outcomes. Nat Rev Drug Discov 10:111-126 